\title{
High-Performance Non-doped Blue OLEDs based on Efficiently Triplet-Triplet Upconversion and Aggregation-induced Emission
}

\author{
Pengbo Han+, Chengwei Lin+, Kaojin Wang, Yanping Qiu, Haozhong Wu, Dongge Ma, ${ }^{*}$ Anjun Qin* and \\ Ben Zhong Tang
}

[a] P. Han, C. Lin, K. Wang, Y. Qiu, H. Wu, Prof. A. Qin, Prof. D. Ma, Prof. B. Z. Tang

State Key Laboratory of Luminescent Materials and Devices, Guangdong Provincial Key Laboratory of Luminescence from Molecular Aggregates, Center for Aggregation-Induced Emission

South China University of Technology

Guangzhou, 510640 (China)

E-mail: msdgma@scut.edu.cn; msqinaj@scut.edu.cn

[b] Prof. B. Z. Tang

Department of Chemistry, Hong Kong Branch of Chinese National Engineering Research Center for Tissue Restoration and Reconstruction

The Hong Kong University of Science and Technology

Clear Water Bay, Kowloon, Hong Kong (China)

Supporting information for this article is given via a link at the end of the document.

\begin{abstract}
Triplet-triplet upconversion (TTU), where two low-energy triplet excitons are converted to one higher energy singlet exciton, is excellent approach to break through the theoretical limit of the pure fluorescent organic light-emitting diodes (OLEDs) by $5 \%$. To data, however, the reported emitters with high emission efficiency and efficiently TTU in film state are rare. Herein, we design the blue aggregation-induced emission luminogens (AIEgens) and investigate their upconversion efficiency. TPA-An-mPhCz can not only achieve high emission efficiency in the film state, but also show high upconversion efficiency of close to $50 \%$ even though the calculated energy level of the triplet excitons $\left(T_{2}\right)$ is lower than $2 T_{1}$. A possible upconversion mechanism is proposed according to the transient electroluminescence spectra and theoretical calculation. This strategy may provide a new platform for the construction of highly efficient nondoped blue OLEDs based on TTU and AIEgens.
\end{abstract}

Organic light-emitting diodes (OLEDs) have been widely used in the field of full-color flat-panel displays and white lighting. ${ }^{[1]}$ In OLEDs, injected electrons and holes recombine to form $25 \%$ singlet excitons yield and $75 \%$ triplet excitons yield. ${ }^{[2]}$ Therefore, one of the most effective methods to enhance the efficiency of OLEDs requires the contribution from triplet excitons. Phosphorescent OLEDs (PhOLEDs) containing transition metal complexes can achieve $100 \%$ internal quantum efficiency (IQE) through strong spin-orbit coupling effect of heavy atom. ${ }^{[3]}$ Alternatively, the lowest triplet $\left(\mathrm{T}_{1}\right)$ excitons can be up-converted to singlet $\left(S_{1}\right)$ ones through reverse intersystem crossing (RISC) process to realize thermally activated delayed fluorescence (TADF) ${ }^{[4]}$, leading to nearly $100 \%$ IQE. ${ }^{[5]}$ Although blue $\mathrm{Ph}$ - and TADF-OLEDs can harvest triplet excitons efficiently, their operation lifetime and efficiency roll-off in high voltage is still a hindrance due to their long exciton lifetime and high triplet energy levels. ${ }^{[6]}$ Low $T_{1}$ exciton, an intermediate, can be converted into high $S_{1}$ state through triplet fusion (TF) process to make use of triplet excitons. ${ }^{[7]}$ Triplet-triplet upconversion (TTU) OLEDs can not only achieve a high IQE, but also possess long operation lifetime compared to blue TADF and Ph-OLEDs. ${ }^{[8]}$ Therefore, it is highly desirable to develop efficient blue TTU-OLEDs.

As shown in Figure 1A, two triplet fusion generates an intermediate state $\left(T_{1}+T_{1}\right)$, whose spin-spin coupling leads to the formation of $1 / 9$ singlet ${ }^{1}(T T), 1 / 3$ triplet ${ }^{3}(T T)$, and $5 / 9$ quintet
${ }^{5}(T T) \cdot{ }^{[9]}$ The ${ }^{1}(T T)$ and ${ }^{3}(T T)$ can form $\mathrm{S}_{1}$ and $\mathrm{T}_{1}$ with a molecular ground state $\left(\mathrm{S}_{0}\right)$, respectively. Conversely, ${ }^{5}(T T)$ could be upconverted to $T_{1}+T_{1}$ intermediate state, because its energy is lower than that of quintet (intramolecular) excited state $\left(Q_{1}\right){ }^{[8 c]}$ Therefore, $T_{1}$ can form $S_{0}$ by the repeated TF process with a upconversion efficiency $\left(\eta_{\text {TTU }}\right)$ of close to $20 \%$. ${ }^{\left[{ }^{[a]}\right]}$ When the triplet states $\left(T_{2}\right)$ energy level is higher than $2 T_{1},{ }^{3}(T T)$ can be expected to return directly to $2 \mathrm{~T}_{1}$, and the $\eta_{\mathrm{TTU}}$ can be also raised to $50 \%$. ${ }^{[10]}$ However, the high TF efficiency is only limited to some special materials, such as an anthracene derivative and rubrene, due to the strict energetic requirements. ${ }^{[8 \mathrm{a}, 11]}$ Alternatively, the transition from ${ }^{3}(T T)$ to $S_{1}$ has been suggested to break through the limit of $\eta_{\text {TTU. }}{ }^{[12]}$ Although it is an potential path to improve $\eta_{\text {TTU }}$, blue OLED with high-performance based on the transitions is rare because of the absence of effective molecular design.

9,10-diphenylanthracene (DPA), a polycyclic aromatic hydrocarbon (PAH) compound with low triplet level and good stability, is typically used as an emitter for construction of blue TTU OLEDs. ${ }^{[13]}$ In principle, TTU is a bimolecular process and needs high enough triplet exciton concentration to improve TF efficiency. However, DPA is usually doped into appropriate host materials due to its aggregation-caused quenching (ACQ) effect. ${ }^{[14]}$ As a result, it is difficult for the doped devices to achieve high-performance blue OLEDs by controlling TF process. More importantly, it is complicated to prepare the doped devices. Therefore, developing $\mathrm{PAH}$ compounds with intense film state emission is beneficial for the construction of high-performance non-doped blue TTU-OLEDs. Exactly opposite to the ACQ effect, the luminogens with aggregation-induced emission (AIE) characteristics can exhibit intense emission in their film state. ${ }^{[15]}$ Thus, they are expected to combine PAH compounds with TTU feature to solve above problems. According to the AIE design concept, new AIE luminogens (AlEgens), decorating ACQ molecules with triphenylamine (TPA) moiety, have also been achieved. ${ }^{[16]}$ In addition, Konishi et.al illustrated that the frontier orbitals of diarylamine and anthracene interpenetrated when the donor plane was distorted ${ }^{[17]}$, meanwhile, a transition between molecular orbitals with relative orthogonal directions might also 


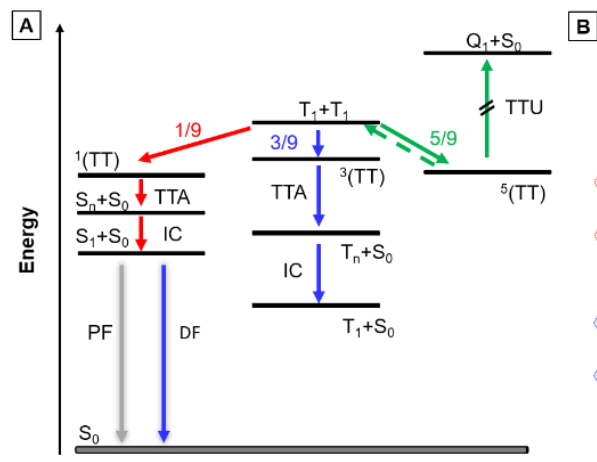

B

c

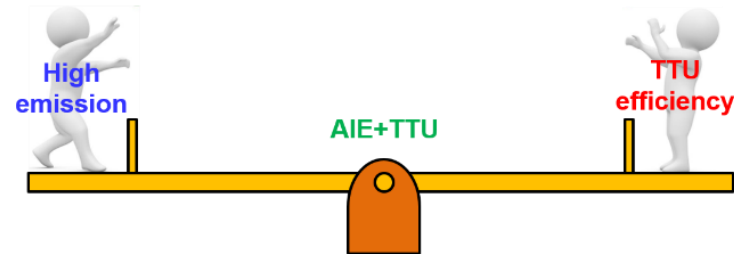

Figure 1. (A) Energy-level diagram illustrating the mechanisms of triplet-triplet upconversion (TTU) and triplet-triplet annihilation (TTA). ${ }^{1}(T T),{ }^{3}(T T)$, and ${ }^{5}(T T)$ are singlet, triplet, and quintet intermediate states. $S_{1}, T_{1}$, and $S_{0}$ are singlet excitons, triplet excitons, and ground states. $S_{n}$ and $T_{n}$ are higher singlet and triplet states. (B) Chemical structures of the anthracene derivatives. (C) Design principle of blue emitters.

promote to the conversion process from ${ }^{3}(T T)$ to $S_{1} \cdot{ }^{[12]}$

Herein, the compounds TPA-An-Ph and TPA-An-mPhCz, in which the distorted donating groups of triphenylamine (TPA) was attached on anthracene (An) cores together with the metapositions substituted plane group of carbazole $(\mathrm{Cz})$ group, were designed and synthesized (Figure 1B). The carbazole group with weaker electron-donating ability can facilitate the hole transport of devices, which in turn improve the device performance. ${ }^{[18]}$ In addition, it was demonstrated that high-efficiency solid-state emission can be achieved by the meta-substituted strategy. ${ }^{[19]}$ Attracted by their AIE feature, TPA-An-Ph and TPA-Ph-mPhCz show high photoluminescence quantum efficiency yields (PLQYs) in their film state. These non-doped blue OLEDs using the AlEgens as emitting layers (EMLs) exhibit low efficiency roll off. The TPA-An-Ph-based OLED gives a maximum forward-viewing external quantum efficiency (EQE) of $4.51 \%$. Notably, the TPAAn-mPhCz-based OLED achieves a maximum EQE of $8.1 \%$. The transient electroluminescence (EL) spectra and theoretical calculation suggest that this is attribute to the effective TTU conversion process from ${ }^{3}(T T)$ to $\mathrm{S}_{n}$ of TPA-An-mPhCz. This strategy may provide an instructive way to achieve highly efficient commercialized blue OLEDs by the construction of AlEgen with effective TTU process (Figure 1C).

The synthetic routes of TPA-An-Ph and TPA-An-mPhCz are shown in Scheme S1. The target products of TPA-An-Ph and TPA-An-mPhCz were facilely synthesized in $80 \%$ yield according to the Suzuki coupling reaction. The structures of TPA-An-Ph and TPA-An-mPhCz were fully characterized by ${ }^{1} \mathrm{H}$ and ${ }^{13} \mathrm{C} N M R$ and high resolution mass (HRMS) spectroscopy. For comparison, the purchased DPA was used to explore the rationality of the design. DPA, TPA-An-Ph, and TPA-An-mPhCz are soluble in commonly used organic solvents, such as dichloromethane (DCM) and tetrahydrofuran (THF), but insoluble in water.

Photo-physical properties of DPA, TPA-An-Ph, and TPA-An$\mathrm{mPhCz}$ were studied. Figure $2 \mathrm{~A}$ shows their ultraviolet-visible (UV-Vis) absorption spectra in THF solutions. The absorption peak with vibronic structures originates from the anthracene core. ${ }^{[20]}$ The PL spectra of DPA, TPA-An-Ph and TPA-An-mPhCz in THF solutions exhibit deep blue and blue emissions with peaks ranging from 409 to $476 \mathrm{~nm}$ upon photoexcitation (Figure S1). In comparison with DPA in THF solution, the emission peaks of TPAAn-Ph and TPA-An-mPhCz were considerably red-shifted.
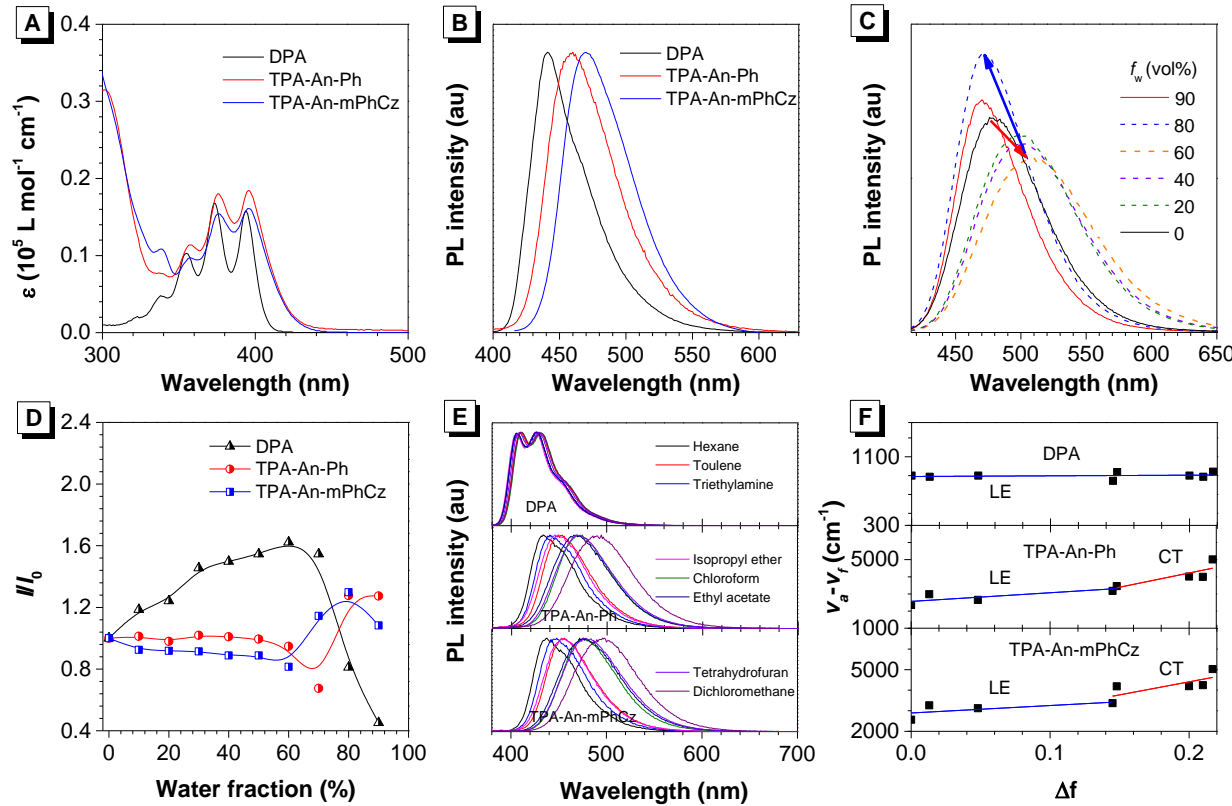

Figure 2. (A) UV-vis (THF solution) and (B) photoluminescence (PL, film state) spectra of DPA, TPA-An-Ph, and TPA-An-mPhCz. (C) PL spectra of TPA-An-mPhCz in THF/water mixtures with different water fractions $\left(f_{\mathrm{w}}\right) ; \lambda_{\mathrm{ex}}: 360 \mathrm{~nm}$; concentration: $10 \mu \mathrm{M}$. (D) Plots of $I / I_{0}$ versus $f_{\mathrm{w}}$, where $I_{0}$ is the PL intensity in pure THF solution. (E) Effects of solvent on fluorescence spectra of the anthracene derivatives. (F) Stokes shifts of the anthracene derivatives as a function of the orientation polarizability. The solid lines are the fitting results by the Lippert-Mataga equation. 
Meanwhile, featureless PL spectra were obtained for TPA-An-Ph and TPA-An-mPhCz in THF solutions, implying that each emission originates from charge transfer (CT) state. In the vacuum-deposited neat film state, the PL spectra of DPA, TPA$\mathrm{An}-\mathrm{Ph}$, and TPA-An-mPhCz films show deep blue and blue regions with peaks at 442,460 and $470 \mathrm{~nm}$, respectively (Figure $2 \mathrm{~B})$. The photoluminescence quantum yields $\left(\Phi_{\mathrm{F}}\right)$ value of DPA in THF solution was $68.1 \%$, while its value was reduced to $59.3 \%$ in vacuum-deposited neat film. Notably, $\Phi_{F}$ values of TPA-An-Ph and TPA-An-mPhCz in THF solutions were measured to be 30.0 and $57.8 \%$, while these values were enhanced to $40.1 \%$ and $65.1 \%$, respectively, in vacuum-deposited neat film. These results suggest that TPA-An-Ph and TPA-An-mPhCz exhibit the aggregation-enhanced emission (AEE) feature.

To further explore the AEE feature of TPA-An-Ph and TPA$\mathrm{An}-\mathrm{mPhCz}$, their $\mathrm{PL}$ behaviors was explored in THF/water mixtures with different water fractions $\left(f_{\mathrm{w}}\right)$. As shown in Figures $2 \mathrm{C}$ and $\mathrm{S} 2$, the emission peak slowly red-shifted along with the decrease in PL intensity when $f_{w}$ was gradually increased, which is attributed to the effect of twisted intermolecular charge transfer (TICT). ${ }^{[21]}$ However, when the water content was further increased their PL intensities gradually increase along with blue-shifted emission peak owing to the formation of aggregates and activation of restriction of intramolecular motion (RIM). ${ }^{[22]}$ In contrast with TPA-An-Ph and TPA-An-mPhCz in the mixtures of THF/water with different $f_{w}$, PL behavior of DPA shows completely opposite trend (Figure 2D), further witnessing the effectiveness of our design strategy.

To concretely analyze excited states of DPA, TPA-An-Ph, and TPA-An-mPhCz, their PL spectra were investigated in different solvents (Figure 2E). According to the Lippert-Mataga model, ${ }^{[23]}$ the dipole moment of the excited state can be acquired by a slope of the Stokes shift $\left(\mathrm{V}_{\mathrm{a}}-\mathrm{V}_{\mathrm{f}}\right)$ as a function of the orientation polarizability (f) as shown in Tables S1-S3. The fitting results are shown in Figure $2 \mathrm{~F}$. The derived dipole moments $\left(\mu_{\mathrm{e}}\right)$ of the excited state of DPA is $0.69 \mathrm{D}$, indicating that its excited state is assigned to localized excited (LE) states. On the other hand, TPA$\mathrm{An}-\mathrm{Ph}$ and TPA-An-mPhCz exhibit the two independent slops: smaller $\mu_{\mathrm{e}}$ values of 7.27 and $9.02 \mathrm{D}$ in low-polarity solvents and larger $\mu_{\mathrm{e}}$ values of 12.93 and $14.95 \mathrm{D}$ in high-polarity solvents, respectively. These results suggest that the excited states are assigned to LE and CT states, respectively. Meanwhile, their PL spectra in film state were measured at $77 \mathrm{~K}$ (Figure S3). The PL peaks of DPA, TPA-An-Ph, and TPA-An-mPhCz are 424 (2.92 $\mathrm{eV}), 454(2.73 \mathrm{eV})$, and $467 \mathrm{~nm}(2.56 \mathrm{eV})$, respectively. The PL spectra of TPA-An-Ph and TPA-An-mPhCz in film state are close to those in polar solvents rather than those in nonpolar solvents. Therefore, the $\mathrm{S}_{1}$ states of TPA-An-Ph and TPA-An-mPhCz films would be assigned to a ${ }^{1} \mathrm{CT}$ mixing weak ${ }^{1} \mathrm{LE}$ state. Moreover, the PL decay curves of DPA, TPA-An-Ph, and TPA-An-mPhCz were plotted in Figure S4. Their lifetime shows a single-exponential fluorescence decay process in THF and solid film, suggesting that they emit fluorescence.

To have a deeper understanding of the structure-property relationships, the single crystal of TPA-An-mPhCz (CCDC 2042010) was obtained by solvent evaporation method and analyzed by X-ray diffraction crystallography. As shown in Figure 3A, TPA-An-mPhCz possesses a highly twisted L-shape molecular configuration with large dihedral angles distributing between $61^{\circ}$ and $81^{\circ}$. Moreover, strong intermolecular $\mathrm{C}-\mathrm{H} \cdots \mathrm{H}-\mathrm{C}$ interference with a short distance of 2.326-2.399 A and multiple

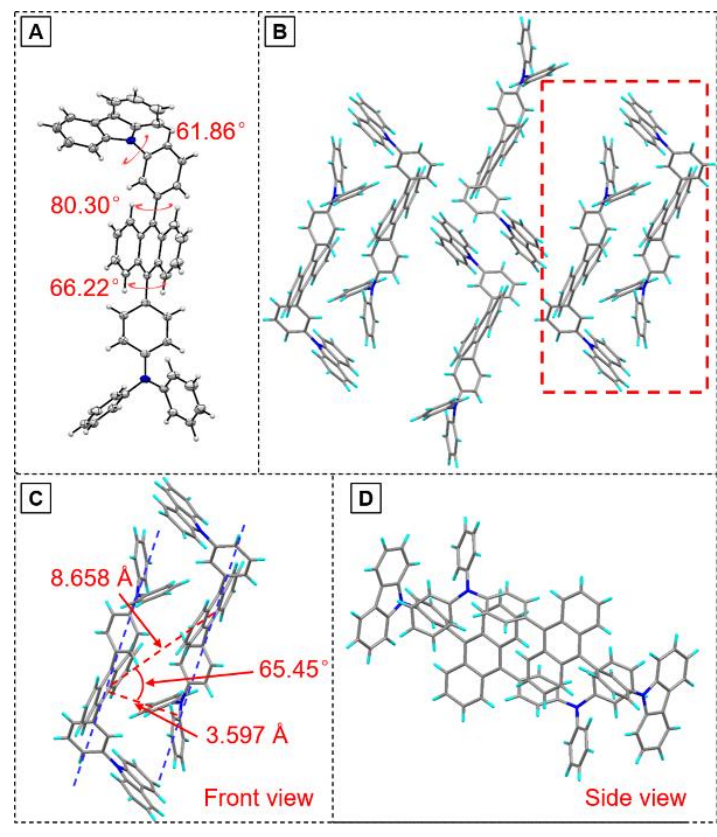

Figure 3. (A) Crystal structure and (B) packing pattern of TPA-An-mPhCz in single crystals; (C) front view and (D) side view of the detailed molecular stacking with red dotted line.

intermolecular $\mathrm{C}-\mathrm{H} \cdots \pi$ hydrogen bonds with distances of 2.702$3.721 \AA$ are found between the molecules (Figure 3B). No close $\pi-\pi$ stacking was found in Figure $3 C$ and $3 D$. These factors can effectively rigidify molecular conformation and reduce nonradiative energy dissipation in the aggregate state, resulting in an AEE effect in the aggregate state. ${ }^{[24]}$

The thermal stabilities of TPA-An-Ph and TPA-An-mPhCz were then investigated by differential scanning calorimetry (DSC) and thermogravimetric (TG) analysis. As depicted in Figure S5 and Table S4, the decomposition temperatures ( $T_{\mathrm{d}}, 5 \%$ weight loss) of TPA-An-Ph and TPB-An-mPhCz are 362 and $411{ }^{\circ} \mathrm{C}$, respectively, but their glass transition temperatures $\left(T_{\mathrm{g}}\right)$ are not found. These results prove that the compounds exhibit good thermal stability, which is suitable for the preparation of vapor deposition device. In addition, the electrochemical properties of TPA-An-Ph and TPA-An-mPhCz were measured by using cyclic voltammetry (CV). As shown in Figure S6, the highest occupied molecular orbital (HOMO) energy levels of TPA-An-Ph and TPA$\mathrm{An}-\mathrm{mPhCz}$ are deduced to be -5.20 and $-5.22 \mathrm{eV}$, respectively, while the lowest unoccupied molecular orbital (LUMO) energy levels of TPA-An-Ph and TPA-An-mPhCz are deduced to be -2.51 and $-2.52 \mathrm{eV}$, respectively, which will facilitate the device configuration optimization.

Thanks to the excellent thermal stability and the high emission efficiency in aggregate state, the non-doped devices were constructed by the use of TPA-An-Ph and TPA-An-mPhCz as EMLs with a configuration of ITO/HAT-CN (5 nm)/TAPC (60 $\mathrm{nm}) / \mathrm{TCTA}(5 \mathrm{~nm}) /$ emitter $(20 \mathrm{~nm}) / \mathrm{TmPyPB}(40 \mathrm{~nm}) / \mathrm{LiF}$ (1 nm)/Al (Figure $4 \mathrm{~A})$, where ITO, 1,4,5,8,9,11hexaazatriphenylenehexacarbonitrile (HAT-CN), 1-bis[4-[N,Ndi(4-tolyl)amino]phenyl]-cyclo-hexane (TAPC), 4,4',4"'-tri(Ncarbazolyl)-triphenylamine (TCTA), and 1,3,5-tri[(3-pyridyl)-phen3-yl]benzene (TmPyPB) act as the anode, hole injection, holetransporting, exciton-blocking layers, and electron-transporting layers, respectively. These non-doped blue OLEDs exhibit 

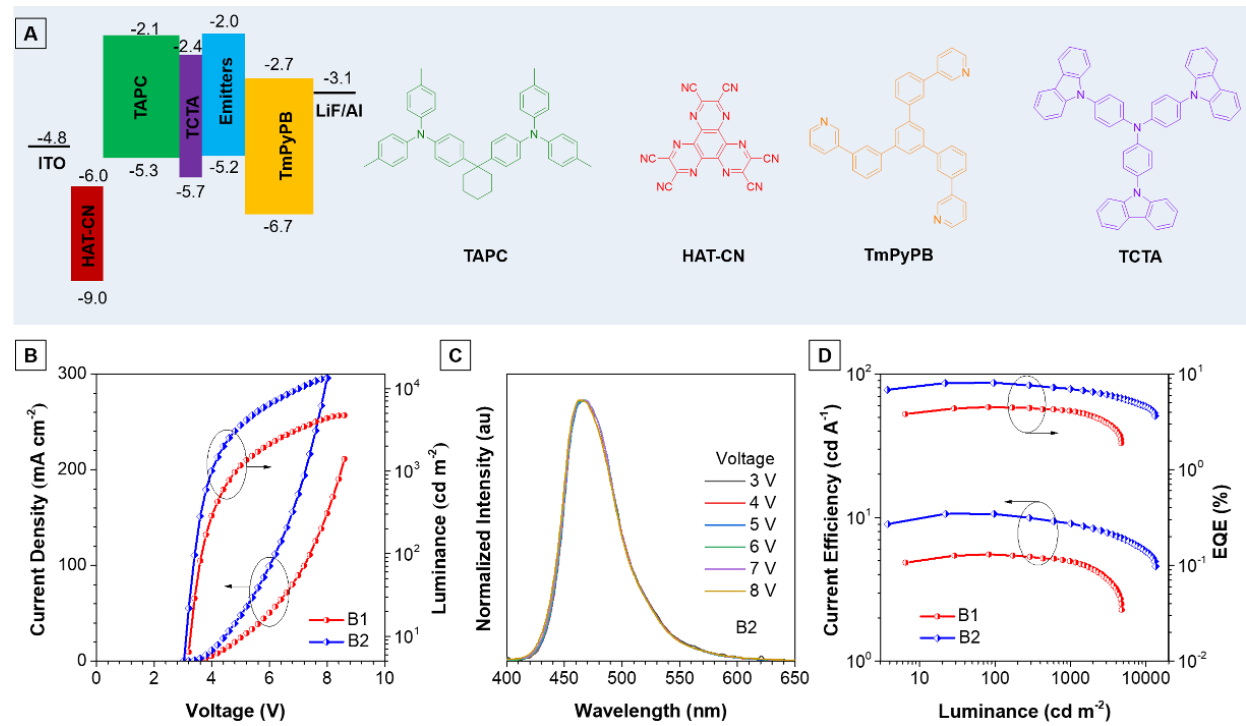

Figure 4. (A) Device structure and ionization potentials (IPCV) and electron affinities (EACV) for each material. (B) Current density-voltage-luminance (J-V-L) characteristics of devices B1 and B2. (C) Electroluminescence (EL) spectra of device B2 at various voltages. (D) CE and EQE versus luminance curves of the nondoped OLEDs based on AIEgens of TPA-An-Ph and TPA-An-mPhCz.

excellent device performance at low operation voltages (Figure 4B). Meanwhile, these EL spectra keep stable when the voltage is increased from 3 to $8 \mathrm{~V}$, confirming their good color stability (Figures 4C and S7). Moreover, TPA-An-mPhCz-based OLED presents an emission peak at $470 \mathrm{~nm}$ with a narrow full width at half-maximum (FWHM) of $58 \mathrm{~nm}$, which is useful to blue emission of device. As depicted in Figure 4D, TPA-An-Ph-based OLED achieves a maximum forward-viewing EQE of $4.51 \%$ and $C E$ of $5.54 \mathrm{~cd} / \mathrm{A}$. Notably, TPA-An-mPhCz-based OLED gives a maximum EQE of $8.10 \%$ and $C E$ of $10.62 \mathrm{~cd} / \mathrm{A}$, which is nearly twice that of TPA-An-Ph-based OLED. More importantly, the EQE and CE of TPA-An-mPhCz-based OLED also remain a high value of $6.97 \%$ and $9.09 \mathrm{~cd} / \mathrm{A}$ at a luminance of $1000 \mathrm{~cd} \mathrm{~m}$, demonstrating the low efficiency roll-off of the device. Meanwhile, the power efficiency of these devices shows identical result (Figure S8 and Table S5).

It is worth noting that the EQE of TPB-An-mPhCz-based OLED breaks through the theoretical limit of the pure fluorescent OLEDs by $5 \%$. Theoretically, the EQE equals to $\eta_{\mathrm{eh}} \times \eta_{\mathrm{PL}} \times \eta_{\text {exciton }} \times \eta_{\text {out }}$, where $\eta_{\text {eh }}$ is the factor of the recombination efficiency of injected holes and electrons (ideally $100 \%$ ), $\eta_{\mathrm{PL}}$ is the absolute PLQYs of emitter $(65.1 \%$ for the vacuum evaporated films of TPA-An$\mathrm{mPhCz}), \eta_{\text {exciton }}$ is the radiative exciton ratio, and $\eta_{\text {out }}$ is the light out-coupling efficiency. We measured angular PL data of TPAAn-mPhCz, but no data were obtained. Therefore, $\eta_{\text {out }}$ is assumed to be $20 \%$. According to the above equation, $\eta_{\text {exciton }}$ of the nondoped OLED is calculated to be $62.2 \%$ by using TPA-An-mPhCz as fluorescent EML, suggestive of the involved triplet excitons in the radiative process. The lifetime of TPA-An-mPhCz shows a single-exponential fluorescence decay process with $3.58 \mathrm{~ns}$ in film state. In addition, the excited state $T_{1}$ was tested in film at $77 \mathrm{~K}$, but no result was obtained. These results indicate a large energy gap between $S_{1}$ and $T_{1}$. Therefore, TADF is impossible for high performance OLEDs. To verify the improved mechanism in EL efficiency of TPB-An-mPhCz-based OLED, transient EL decay of the non-doped device at different voltages were analyzed. As shown in Figure $5 \mathrm{~A}$, the EL decay exhibits two parts, the prompt fluorescence and the delayed fluorescence. Triplet excitons return directly to higher excited singlet states, which results in no delayed component in transient EL spectra of hybridized local and charge transfer (HLCT)- OLEDs. ${ }^{[23]}$ Thus, the emission mechanism should be TTU. The prompt part originates from singlet exciton emission immediately under electrical pumping, and the following delayed component is attributed to triplet excitons by the TF process. Moreover, the ratio of delayed fluorescence slowly decreases with increasing voltage. It is a hypothesis that the delayed portion is not simply from the recombination of the trapped charges. ${ }^{[11 b]}$
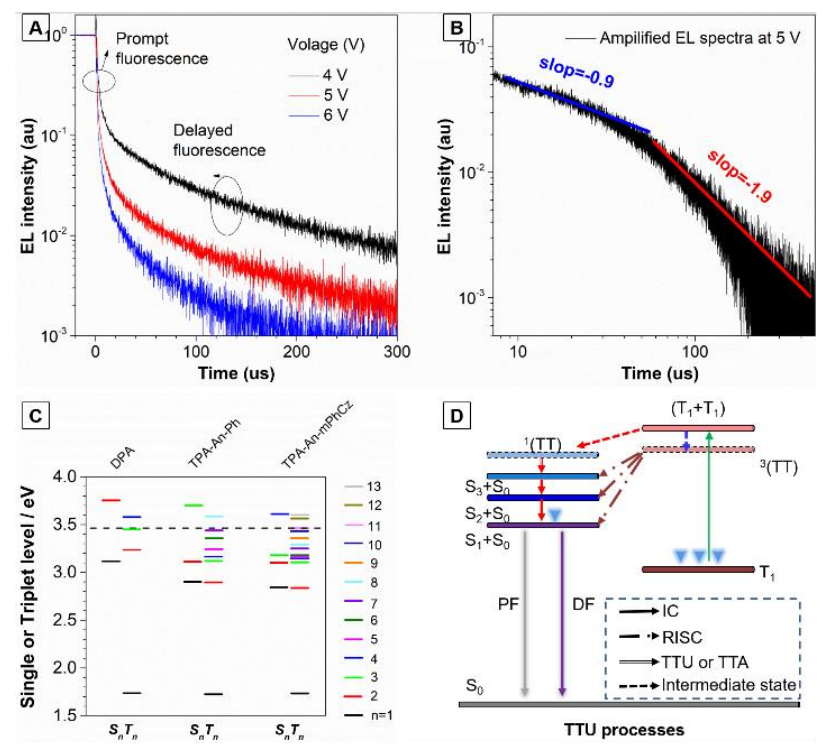

Figure 5. (A) Transient EL decay of the non-doped device B2 at different voltages. (B) Amplified EL spectra of the non-doped device B2 at $5 \mathrm{~V}$. (C) Energy diagrams. Energy levels and twice the $\mathrm{T}_{1}$ energies (dashed line) of the anthracene derivatives calculated using B3LYP/6-311G(d,p), where $n$ is the quantum number used to indicate the excited state. (D) Proposed TTU mechanism based on the emitters. 
As reported by $\mathrm{D}$. Kondakov, the delayed $\mathrm{EL}$ is proportional to $t^{2}$ ( $t$ is the time) when TTU is predominant in OLED. ${ }^{[25]}$ The plot of $\log (E L$ intensity) versus $\log (t)$ for device B2 is shown in Figure $5 \mathrm{~B}$. The slope of the obtained curve is nearly -2 for TTU mechanism. The fitting results are consistent with TTU-related triplet exciton dynamics as described above, further confirming the validity of TTU process in TPB-An-mPhCz-based OLED.

To further confirm our assumption, the excited states of DPA, TPA-An-Ph, and TPA-An-mPhCz were calculated by TD-DFT calculations based on B3LYP/6-311(d,p) basis set. As shown in Figure $5 \mathrm{C}$, the $\mathrm{T}_{1}$ energy level of DPA, TPA-An-Ph and TPA-An$\mathrm{mPhCz}$ is close to $1.73 \mathrm{eV}$, which is consistent with the results reported in the literature. ${ }^{[26]} \mathrm{T}_{1}$ states of DPA, TPA-An-Ph and TPA-An-mPhCz were derived from the ${ }^{3} \mathrm{LE}$ state of the anthracene unit. Thus, their ${ }^{3}(T T)$ are almost identical. The energy of $2 \mathrm{~T}_{1}$ is higher than $\mathrm{T}_{2}$, indicating that $\eta_{\mathrm{TTU}}$ is close to $20 \%$, which in turn leads to $\eta_{\text {exciton }}$ of $32.5 \%$. However, the scenario cannot explain the maximum EQE of TPA-An-mPhCz based OLED. Therefore, the possibility of a conversion process from ${ }^{3}(T T)$ to $S_{n}$ are also discussed. According to the previous work ${ }^{[12]}$, the energy level of $T_{m}$ should be close to that of ${ }^{3}(T T)$. Table $S 6$ lists the electronic transitions related to $T_{m}$ with energies in the range of 3.40-3.50 eV. TTU transition would form $S_{n}$ and then return to $S_{1}$ by internal conversion. Thus, the spin-orbit interaction between $S_{n}$ and $T_{m}$ will be considered. As shown in Table S6, the electronic transitions related to $S_{n}$ are also listed. The possible TTU transition from ${ }^{3}(T T)$ to $S_{n}$ are given in Table S7. For TPA-An-Ph (Figure S9) and TPA-An-mPhCz (Figure S10), TTU transition involves different vectors of the magnetic moments. For example, in TPA-An-mPhCz, as depicted in Figure S10 and Table S7, the spin-orbit matrix of $<\mathrm{S}_{3}\left|H_{s o}\right| \mathrm{T}_{10}>$ consisting of HOMO-2 and HOMO-4 are involved in the TTU transition from ${ }^{3}(T T)$ to $S_{n}$. The HOMO-4 is localized on the anthracene unit, whereas HOMO-2 is mainly localized on the carbazole unit, which is oriented perpendicular to the anthracene unit. As a result, the rotation of the molecular orbitals from the carbazole unit to the anthracene unit is involved in the spin-orbit matrices, leading to TTU process according to spin conversion, as illustrated in Figure 4D. This process is different from HLCT and "Hot" exciton process because the observed delayed fluorescence in OLEDs is derived from the TTU process.

In summary, novel blue AlEgen of TPA-An-mPhCZ, in which TPA-An-Ph is an AIEgen, and meta-substituted carbazole act as electron donating groups, respectively, was designed and synthesized. TPA-An-Ph and TPA-An-mPhCz show excellent thermal stability and high PLQYs in their film state. The nondoped device exhibits excellent device performance. The maximum CE and EQE of TPA-An-mPhCz-based OLED can reach up to $10.62 \mathrm{~cd} / \mathrm{A}$ and $8.10 \%$, respectively, with an operation voltage as low as $3.0 \mathrm{~V}$. More importantly, the CE and EQE still remain a high value of $9.09 \mathrm{~cd} / \mathrm{A}$ and $6.97 \%$, respectively, at a luminance of $1000 \mathrm{~cd} \mathrm{~m}^{-2}$, suggesting low efficiency roll-off of the device. The transient EL spectra and theoretical calculation confirm that the unique TTU process of the emitter plays a crucial role in high EQE. The design idea for the novel triplet-triplet upconversion and aggregation-induced emission is valuable for the construction of high-performance OLEDs, which may be beneficial for practical applications, such as white lighting and full color flat-panel displays.

\section{Acknowledgements}

This work was financially supported by the National Natural Science Foundation of China (21788102 and 21525417), the Natural Science Foundation of Guangdong Province (2019B030301003 and 2016A030312002), and the Innovation and Technology Commission of Hong Kong (ITC-CNERC14S01).

Keywords: aggregation-induced emission $•$ anthracene $\bullet$ triplettriplet upconversion $\bullet$ blue OLEDs $\bullet$ non-doped devices

[1] a) C. W. Tang, S. A. VanSlyke, Appl. Phys. Lett. 1987, 51, 913-915; b) J. Kido, M. Kimura, K. Nagai, Science 1995, 267, 1332-1334; c) Y. Sun, N. C. Giebink, H. Kanno, B. Ma, M. E. Thompson, S. R. Forrest, Nature 2006, 440, 908-912.

[2] W. Helfrich, W. G. Schneider, J. Chem. Phys. 1966, 44, 2902-2909.

[3] a) H. Kuo, Y. Chen, L. R. Devereux, C. Wu, M. A. Fox, C. Kuei, Y. Chi, G. Lee, Adv. Mater. 2017, 29, 1702464; b) C. Adachi, M. A. Baldo, M. E. Thompson, S. R. Forrest, J Appl. Phys. 2001, 90, 5048-5051.

[4] A. Endo, M. Ogasawara, A. Takahashi, D. Yokoyama, Y. Kato, C. Adachi, Adv. Mater. 2009, 21, 4802-4806.

[5] H. Uoyama, K. Goushi, K. Shizu, H. Nomura, C. Adachi, Nature 2012, 492, 234-238.

[6] J. U. Kim, I. S. Park, C. Chan, M. Tanaka, Y. Tsuchiya, H. Nakanotani, C. Adachi, Nat. Commun. 2020, 11, 1-8; b) K. Tuong Ly, R. Chen-Cheng H. Lin, Y. Shiau, S. Liu, P. Chou, C. Tsao, Y. Huang, Y. Chi, Nat. Photonics 2017, 11, 63-68; c) J. Lee, C. Chen, P. Lee, H. Lin, M. Leung T. Chiu, C. Lin, J. Mater. Chem. C 2019, 7, 5874-5888.

[7] a) C. Ganzorig, M. Fujihira, Appl. Phys. Lett. 2002, 81, 3137-3139; b) D. Y. Kondakov, T. D. Pawlik, T. K. Hatwar, J. P. Spindler, J. Appl. Phys. 2009, 106, 124510.

[8] a) A. Salehi, C. Dong, D. Shin, L. Zhu, C. Papa, A. Thy Bui, F. N Castellano, F. So, Nat. Commun. 2019, 10, 1-9; b) J. Huh, Y. H. Ha, S. Kwon, Y. Kim, J. Kim, ACS Appl. Mater. Inter. 2020, 12, 15422-15429; c) X. Qiao, D. Ma, Materi. Sci. Eng. R 2020, 139, 100519.

[9] a) C. Chiang, A. Kimyonok, M. K. Etherington, G. C. Griffiths, V. Jankus F. Turksoy, A. P. Monkman, Adv. Funct. Mater. 2013, 23, 739-746; b) T. L. Keevers, D. R. McCamey, Phys. Rev. B 2016, 93, 045210; c) B. H. Wallikewitz, D. Kabra, S. Gélinas, R. H. Friend, Phys. Rev. B 2012, 85, 045209.

[10] Y. Y. Cheng, T. Khoury, R. G. C. R. Clady, M. J. Y. Tayebjee, N. J. EkinsDaukes, M. J. Crossley, T. W. Schmidt, Phys. Chem. Chem. Phys. 2010, 12, 66-71.

[11] a) D. Di, L. Yang, J. M. Richter, L. Meraldi, R. M. Altamimi, A. Y. Alyamani, D. Credgington, K. P. Musselman, J. L. MacManus-Driscoll, R. H. Friend, Adv. Mater. 2017, 29, 1605987; b) W. Liu, S. Ying, R. Guo, X. Qiao, P Leng, Q. Zhang, Y. Wang, D. Ma, Lei Wang. J. Mater. Chem. C 2019, 7, 1014-1021.

[12] R. leuji, K. Goushi, C. Adachi, Nat. Commun. 2019, 10, 1-10.

[13] R. Sato, H. Kitoh-Nishioka, K. Kamada, T. Mizokuro, K. Kobayashi, Y. Shigeta, J. Phys. Chem. C 2018, 122, 5334-5340.

[14] J. Yang, Z. Chi, W. Zhu, B. Z. Tang, Z. Li, Sci. China. Chem. 2019, 62, 1090-1908.

[15] Z. Zhao, H. Zhang, J. W. Y. Lam, B. Z. Tang, Angew. Chem. Int. Ed. 2020, 59, 2-22

[16] J. Mei, N. L. C. Leung, R. T. K. Kwok, J. W. Y. Lam, B. Z. Tang, Chem Rev. 2015, 115, 11718-11940.

[17] S. Sasaki, S. Suzuki, W. M. C. Sameera, K. Igawa, K. Morokuma, G. Konishi, J. Am. Chem. Soc. 2016, 138, 8194-8206.

[18] P. Han, C. Lin, D. Ma, A. Qin, B. Z. Tang, ACS Appl. Mater. Inter. 2020, 12, 46366-46372

[19] H. Liu, D. Cong, B. Li, L. Ye, Y. Ge, X. Tang, Y. Shen, Y. Wen, J. Wang, C. Zhou, B. Yang, Cryst. Growth Des. 2017, 17, 2945-2949.

[20] X. Tang, Q. Bai, T. Shan, J. Li, Y. Gao, F. Liu, H. Liu, Q. Peng, B. Yang, F. Li, P. Lu, Adv. Funct. Mater. 2018, 28, 1705813 
[21] R. Hu, E. Lager, A. Aguilar-Aguilar, J. Liu, J. W. Y. Lam, H. H. Y. Sung, I. D. Williams, Y. Zhong, K. S. Wong, E. Peña-Cabrera, B. Z. Tang, J. Phys. Chem. C 2009, 113, 15845-15853.

[22] H. Nie, K. Hu, Y. Cai, Q. Peng, Z. Zhao, R. Hu, J. Chen, S. Su, A. Qin, B. Z. Tang, Mater. Chem. Front. 2017, 1, 1125-1129.

[23] W. Li, Y. Pan, L. Yao, H. Liu, S. Zhang, C. Wang, F. Shen, P. Lu, B. Yang, Y. Ma, Adv. Opt. Mater. 2014, 2, 892-901.

[24] J. Guo, X. Li, H. Nie, W. Luo, S. Gan, S. Hu, R. Hu, A. Qin, Z. Zhao, S. Su, B. Z. Tang, Adv. Funct. Mater. 2017, 27, 1606458.

[25] D. Y. Kondakov, J. Appl. Phys. 2007, 102, 114504.

[26] S. Reineke, M. A. Baldo, Sci. Rip. 2014, 4, 3797. 


\section{Entry for the Table of Contents}

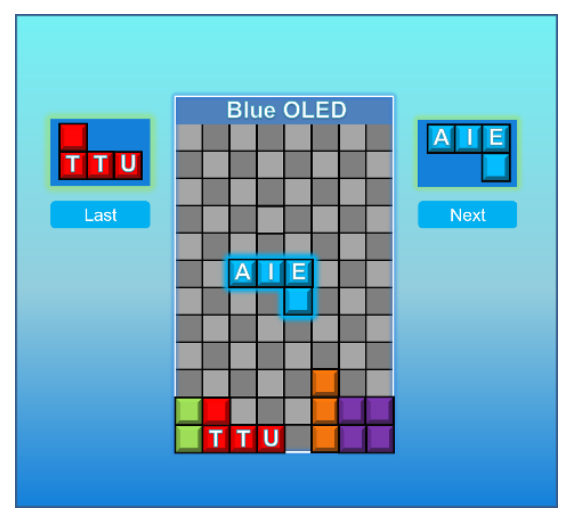

An effective triplet-triplet upconversion (TTU) emitter with aggregation-induced emission (AIE) feature was reported. Thanks to its high solid emission efficiency and effective TTU conversion process from ${ }^{3}(T T)$ to $S_{n}$, the TPA-An-mPhCz-based non-doped OLED realizes an external quantum efficiency as high as $8.10 \%$. The strategy provides a new platform for the construction of highly efficient commercialized blue OLEDs based on TTU and AIE. 